\title{
Effects of chiropractic spinal manipulation on laser-evoked pain and brain activity
}

\author{
Benjamin Provencher ${ }^{1,2}$, Stéphane Northon ${ }^{1,2}$, Carlos Gevers Montoro ${ }^{1,2,3}$, Julie O'Shaughnessy ${ }^{4}$ and \\ Mathieu Piché1, $2^{*}$ (D)
}

\begin{abstract}
The aim of this study was to examine the mechanisms underlying hypoalgesia induced by spinal manipulation (SM). Eighty-two healthy volunteers were assigned to one of the four intervention groups: no intervention, SM at T4 (homosegmental to pain), SM at T8 (heterosegmental to pain) or light mechanical stimulus at T4 (placebo). Eighty laser stimuli were applied on back skin at T4 to evoke pain and brain activity related to $A \delta$ - and $C$-fibers activation. The intervention was performed after 40 stimuli. Laser pain was decreased by SM at T4 $(p=0.028)$ but not T8 $(p=0.13)$, compared with placebo. However, brain activity related to A $\delta$-fibers activation was not significantly modulated (all $p>0.05$ ), while C-fiber activity could not be measured reliably. This indicates that SM produces segmental hypoalgesia through inhibition of nociceptive processes that are independent of A $\delta$ fibers. It remains to be clarified whether the effect is mediated by the inhibition of C-fiber activity.
\end{abstract}

Keywords: Spinal manipulation, Hypoalgesia, Nociceptive fibers, Electroencephalography

\section{Background}

Low back pain and neck pain are among the leading causes of years lived with disability [35]. Recent clinical practice guidelines for these spinal disorders recommend self-management as well as physical and psychological therapies as first-line treatment, while pharmacotherapy and surgery are recommended when first-line treatments were ineffective [13, 21, 37, 58, 62, 63]. Currently, spinal manipulative therapy (SMT) is recommended for the management of spine pain by most of these clinical practice guidelines $[12,13,21,37,58,62]$. However, the neurophysiological mechanisms underlying the painrelieving effects of SMT are still unclear. The understanding of these mechanisms could improve clinical practice by providing optimal conditions in which SMT is most likely to provide pain relief.

*Correspondence: mathieu.piche@uqtr.ca

${ }^{1}$ Department of Anatomy, Université du Québec à Trois-Rivières, 3351 boul. des Forges, C.P. 500, Trois-Rivières, QC G9A 5H7, Canada

Full list of author information is available at the end of the article
The mechanisms by which SMT may relieve pain have been examined in previous studies and reviewed recently $[4,27]$. One potential mechanism is the inhibition of neural processes underlying temporal summation of pain (TSP), the perceptual correlate of wind-up [29]. Wind-up is an increased excitability of dorsal horn neurons caused by repetitive stimulation of afferent $\mathrm{C}$-fibers [29]. It is thought to share common mechanisms with central sensitization and hyperalgesia [1,29], making it a relevant process for the investigation of pain relief by SMT. Decreased TSP on the leg (lumbar dermatome) was reported following lumbar SMT [25]. These results were later replicated and were shown to be specific to spinal manipulation $(\mathrm{SM})$ in healthy volunteers and patients with low back pain (LBP) [6-8]. In contrast, no reduction in TSP was observed between sham and SMT in patients with LBP [2]. However, procedures used in this study were different from previous studies so discrepancies may be explained by methodological differences. In line with the inhibition of C-fiber-related pain by SM, it was shown that TSP produced by repeated electrical 
stimulation in the back is inhibited by SM, while pain produced by a single electrical pulse is not [60].

Altogether, these studies suggest that SM inhibits nociceptive processes related to C-fiber activation, while those related to $A \delta$-fiber activation are unaffected. However, this remains to be examined with neurophysiological methods that allow selective activation of nociceptive fibers and measurement of their activity, since behavioral methods alone are not sufficient for this purpose.

The aim of this study was to determine how brain activity associated with the activation of nociceptive fibers is modulated by SM. Based on the behavioral results of previous studies $[6-8,25,60]$, we hypothesized that pain and pain-related brain activity related to $C$ but not A- $\delta$ fibers would be inhibited by SM.

\section{Methods}

\section{Experimental design}

This study relied on a mixed design to compare changes in laser-evoked pain and brain activity between four groups. A random-number generator was used to create a randomization sequence and assign participants to one of the four experimental groups: no intervention $(n=20)$, placebo intervention (light mechanical stimulus segmental to laser-evoked pain; $n=21$ ), SM segmental to laserevoked pain (SM-T4: $n=21)$ and SM heterosegmental to laser-evoked pain (SM-T8: $n=20)$.

Choosing a placebo intervention for spinal manipulation is challenging. No placebo intervention can account for all aspects of SM [57]. A commonly used placebo intervention consists of a skin contact with no thrust, or soft pressing [57]. The intervention aims at reproducing the spinal manipulation set up and the contact with the participant. In the present study, we selected this intervention as placebo intervention and skin contact was achieved with a hand-held dynamometer to standardize the applied force. This procedure is identical to that used in a previous study [60]. In addition to the placebo group, we included a control group to measure non-specific temporal effects, in which no intervention was applied.

\section{Participants}

A flowchart detailing the participants inclusion in the study and analyses is presented in Fig. 1. Eightytwo healthy volunteers ( 40 men and 42 women; aged $26.6 \pm 7.8$ years [mean $\pm S D$ ]) were recruited by advertisement on the campus of Université du Québec à TroisRivières. Participants were included if they were between 18 and 55 years old. They were excluded if they reported acute or chronic pain, acute or chronic illness, psychiatric disorders, if they underwent spinal surgery, or took any medication or recreational drugs during the 2 weeks prior to experimentation.

\section{Experimental protocol}

During the experiment, room temperature was kept constant at $24^{\circ} \mathrm{C}$ while participants comfortably lay prone on a chiropractic table. Their head was slightly elevated by a folded towel placed under their chin to avoid putting pressure on the electrooculography (EOG) and frontal electrodes. The participant and experimenter wore safety glasses designed for a 1340-nm wavelength laser. Participants were instructed to keep their eyes open, look at a fixation cross to minimize eye movement and refrain movement as much as possible during stimulation. The experiment comprised 80 laser stimuli delivered with an inter-stimulus interval that varied between 8 and $10 \mathrm{~s}$. After each set of 20 stimuli, participants provided pain ratings and could blink as needed during a pause of $60 \mathrm{~s}$. The intervention (SM or placebo) was performed after 40 stimuli, which were used as baseline for data analyses (see PRE condition in Fig. 2).

\section{Laser stimulation}

Painful stimuli were produced by laser heat pulses generated by an infrared neodymium-doped yttrium aluminum perovskite laser (Nd:YAP, DEKA 1340; Electronical Engineering, Florence, Italy). These stimuli have been shown to activate nociceptors selectively $[11,55]$. The stimulation protocol was identical to the protocol used in a previous study reporting an increased ability to detect C-fiber laser-evoked potentials [31], except for the target (back instead of hand dorsum) and the smaller number of stimuli. The laser beam was transmitted through a $10-\mathrm{m}$ optic fiber cable. Laser pulse duration was set to $5 \mathrm{~ms}$ and laser beam to $7 \mathrm{~mm}\left(\approx 38.5 \mathrm{~mm}^{2}\right.$ area). Based on safety recommendations for repeated laser stimuli [40], a maximum fluence limit was set to $14 \mathrm{~J} / \mathrm{cm}^{2}$ (5.25 J intensity limit for a $7 \mathrm{~mm}$ diameter). The laser was triggered using a stimulus presentation software (Spike2; Cambridge Electronic Design Limited, Cambridge, UK). To avoid stimulation of the same area more than once per block, 25 ink marks were drawn on the area to be stimulated in the back with a regular $\mathrm{Hi}$ Tecpoint 0.5-mm Black Pilot pen, in a $5 \times 5 \mathrm{~cm}$ grid centered around $\mathrm{T} 4$ spinous process (T4-T5 dermatome). The laser stimuli were targeting the marks, but the diameter of the laser beam far exceeded the size of the marks. The laser was moved to the next point of the grid after each stimulus. This procedure is safe for experimentation using Nd:YAP laser [40].

Individual pain threshold was determined using a staircase procedure. Before pain threshold assessment, participants were instructed to focus on the warm/burning sensation in their back and to report pain intensity verbally after each stimulus using a numerical rating scale 


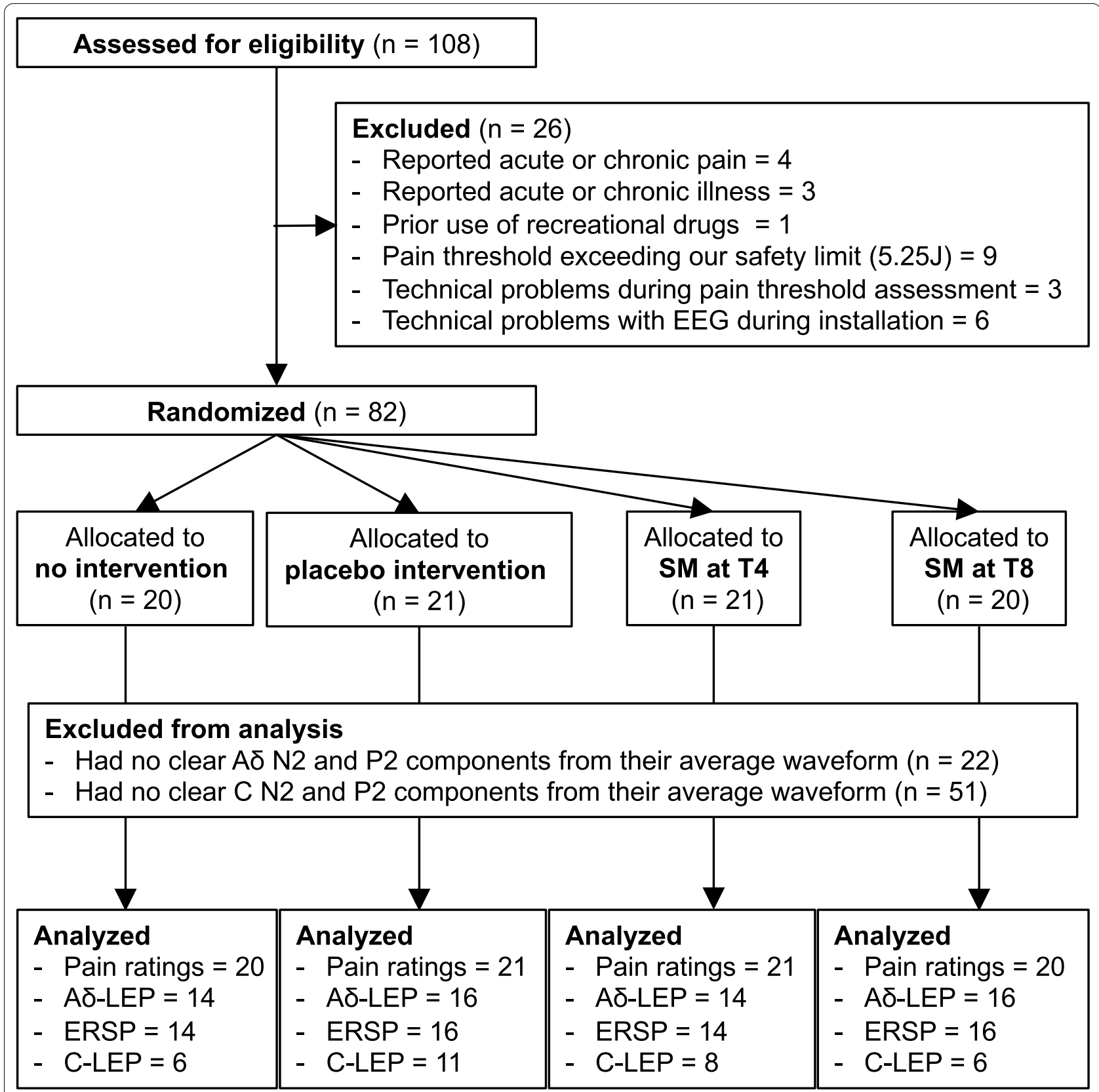

Fig. 1 Flow diagram of enrollment, allocation, and analysis. SM spinal manipulation; LEP laser-evoked potential, ERSP event-related spectral perturbations

ranging from 0 to 100,0 indicating "no pain" and 100 "the worst pain imaginable". Stimuli were delivered at an initial intensity of $0.5 \mathrm{~J}$ and stimulus intensity increased sequentially by $0.5 \mathrm{~J}$ increments until pain was reported (rating of $1 / 100$ or higher), or until the $5.25 \mathrm{~J}$ safety limit was reached. If no pain was reported (rating of 0/100) at the highest energy laser stimulus within our safety limits $(5.25 \mathrm{~J})$, the participant was excluded from the study. This is necessary for the purpose of the study, in which we examine pain inhibition and not only LEPs. Nine participants were excluded for this reason. Otherwise, the energy was increased sequentially again until a pain rating of at least 30/100 was reported or until the $5.25 \mathrm{~J}$ limit was reached. Participants were then familiarized with the selected intensity using five consecutive stimuli with an inter-stimulus interval varying between 5 and $10 \mathrm{~s}$. If the intensity was deemed acceptable for the participant, the experiment was continued. If the participant judged 


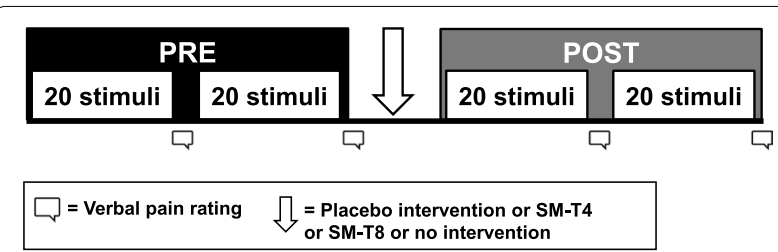

Fig. 2 Experimental paradigm. Participants received four blocks of 20 laser stimuli for a total of 80 stimuli. After each block, participants were instructed to rate pain verbally. Between block 2 and 3 , participants received either no intervention, the placebo intervention, spinal manipulation at T4 (SM-T4) or spinal manipulation at T8

(SM-T8). Laser-evoked pain and brain activity were averaged over 40 stimuli and were compared before and after the intervention (PRE vs POST conditions)

that the stimulus intensity produced pain that could not be tolerated for the duration of the experiment, stimulus intensity was decreased by $0.5 \mathrm{~J}$ and the familiarization procedure resumed until an acceptable stimulus intensity was reached. Once approved by the participant, the stimulus intensity was kept constant throughout the experiment.

\section{Spinal manipulation and placebo intervention}

A previous study has shown that hypoalgesic effects can be produced by a SM at T4, where pain was applied (segmental SM) [60]. In the present study, T4 and T8 segments were selected for segmental and heterosegmental $\mathrm{SM}$, for this reason and for practical reasons; with spinal manipulation at these segments, the participant can lie down comfortably without moving for the intervention. This allows artifact-free recording of brain activity. SM were performed by a licensed chiropractor and consisted in a short-duration, high-velocity, low-amplitude, posterior-to-anterior thrust applied with both hands. SM was applied over the transverse processes of $\mathrm{T} 4$ or $\mathrm{T} 8$ vertebrae to generate audible release (cavitation). This type of manipulation lasts less than $200 \mathrm{~ms}$ and involves a force of approximately $500 \mathrm{~N}$ [30, 64]. Immediately after receiving SM, participants were asked to report if the intervention was painful. No pain was reported in $95 \%$ (39/41) of participants. In two participants, light pain was reported only for the short thrust phase. The placebo intervention consisted of a $20-\mathrm{N}$ force applied for $2 \mathrm{~s}$ over the spinous process of $\mathrm{T} 4$ using a hand-held dynamometer (Hoggan scientific LLC, model Micro FET2, Salt Lake City, UT, USA).

It is not possible to make participants blind to the intervention in the case of spinal manipulation. However, participants were not informed about where the intervention (SM or placebo) was delivered, that other participants would receive different interventions and what was the effect expected in this experiment. No adverse event was reported by participants following SM or the placebo intervention.

\section{Pain ratings}

After each series of 20 stimuli, participants were instructed to rate pain verbally using a numerical rating scale ranging from 0 to 100,0 indicating "no pain" and 100 "worse pain imaginable". They were instructed to report the average pain induced by the 20 stimuli.

\section{Expectations of pain modulation}

Expectations of pain modulation were measured using a visual analogue scale [26]. Before the experiment, participants were presented a form with the following question: "On the scale below, indicate the change in laser-pain intensity that you expect following the intervention in your back". The scale was a horizontal line ranging from -100 to 100 with the following anchors: $-100=$ "maximum decrease", $0=$ "no change" and $100=$ "maximum increase".

\section{Electroencephalographic recordings}

Electroencephalography (EEG) was recorded using a 64-channel BrainVision system with active $\mathrm{Ag}-\mathrm{AgCl}$ electrodes mounted on an actiCAP according to the International 10-20 system (Brain Products, Gilching, Germany). Electrodes were nose-referenced, and the ground was set at FPz. Signals were sampled at $1000 \mathrm{~Hz}$ and filtered using a $0.01-100 \mathrm{~Hz}$ band-pass filter. Eye movements and blinks were recorded using right eye EOG with electrodes placed at the suborbital ridge and just lateral to the external ocular canthus. Electrodes impedance was kept below $20 \mathrm{k} \Omega$.

\section{Laser-evoked potentials (LEP) analysis}

EEG signals were analyzed offline using EEGLAB v.13.5.4b [19]. After applying a $0.5-30 \mathrm{~Hz}$ finite impulse response (FIR) band-pass filter and re-referencing to the common average, data were segmented into epochs extending from $-100 \mathrm{~ms}$ to $+1500 \mathrm{~ms}$ relative to stimulus onset. Epochs were baseline corrected using the -100 to $0 \mathrm{~ms}$ window and then visually inspected to reject those most likely containing artifacts (amplitude value exceeding $\pm 100 \mu \mathrm{V}$ ). On average $3.2 \pm 2.4$ out of 80 epochs (4\%) were rejected. After, an Infomax-independent component analysis (ICA) was applied using the inbuilt EEGLAB function Runica to identify and remove components associated with noise (e.g., eye movement, eye blinks, cardiac, and muscle artifacts).

Finally, average waveforms were computed for each participant and condition. LEP components of interest, including the N2 and P2 [31, 36, 51, 61] were extracted 
from these waveforms. From the 82 participants tested, $22(26.8 \%)$ did not have clear $\mathrm{A} \delta$-N2 and $\mathrm{A} \delta$-P2 peaks and $51(62.2 \%)$ did not have clear C-N2 and C-P2 peaks from their average waveforms. This was assessed independently by three of the experimenters (BP, SN and MP). The N2 and P2 calculations were thus performed on data from the remaining 60 participants for $A \delta$-fiber LEP and on data from the remaining 31 participants for $\mathrm{C}$-fiber LEP (see Fig. 1 for the distribution of these participants among experimental groups). The $\mathrm{A} \delta-\mathrm{N} 2$ was defined as the first major negative deflection occurring between 170 and 400 ms with a maximum amplitude at the vertex $(\mathrm{Cz})$, and the $\mathrm{A} \delta-\mathrm{P} 2$ was defined as the first major positive deflection occurring between 280 and 500 ms with a maximum amplitude at the vertex $(\mathrm{Cz})$. The $\mathrm{C}-\mathrm{N} 2$ was defined as the first major negative deflection occurring between 450 and $600 \mathrm{~ms}$ with a maximum amplitude at the vertex $(\mathrm{Cz})$ that followed the $\mathrm{A} \delta-\mathrm{P} 2$, and the C-P2 was defined as the first major positive deflection occurring between 550 and $800 \mathrm{~ms}$ with a maximum amplitude at the vertex $(\mathrm{Cz})$ that followed the $\mathrm{C}-\mathrm{N} 2$. Latencies vary depending on stimulus location. For the back, the latency of A-delta fibers LEP remains the same regardless of the spinal level stimulated (from C5 to L5) [16, 22, 34]. However, the latencies of C-fibers LEP vary depending on the distance between the stimulus location and the brain [34, 59]. The latencies reported for C-fibers LEP in the present study are consistent with previous findings for stimuli applied to the upper back (T2, T4, T6, T8) [34, 59]. Besides, peak amplitude was calculated for each component instead of peak-to-peak, since each peak originates from different brain generators and reflects distinct neural processes $[24,43,46]$.

\section{Event-related spectral perturbations analysis}

Event-related spectral perturbations (ERSP) [52] were examined for two reasons. Firstly, since nociceptive C-fibers have a largely variable response latency, averaging multiple trials to obtain laser-evoked potentials reduces amplitude [31, 39]. Accordingly, time-domain analyses only give partial access to the evoked brain activity [45]. Therefore, LEP and ERSP analyses are complementary and allow the measurement of brain responses that are phase-locked to stimulus onset or not. ERSP were used in previous studies investigating C-fibers laser-evoked brain activity [20,31,36,44]. Secondly, we were interested in measuring gamma-band oscillations for their potential as a reliable biomarker of nociception and pain $[28,56]$.

ERSP were analyzed with EEGLAB. After applying a 1-100 Hz FIR band-pass filter and re-referencing to the common average, data were segmented into epochs extending from $-2000 \mathrm{~ms}$ to $+2000 \mathrm{~ms}$ relative to stimulus onset. Epochs were baseline corrected using the
$-700--200 \mathrm{~ms}$ window and, as described above, visual inspection and ICA were applied to remove artifacts. A Morlet wavelet convolution [45] was computed using the channel time-frequency options available in EEGLAB v.13.5.4b [19]. Two hundred time points were generated, and 100 linearly spaced frequencies were computed from 1 to $100 \mathrm{~Hz}$. Variable cycles were used for low and high frequencies, with 3 cycles for lowest frequencies and up to 15 cycles for highest frequencies [19]. This variable number of cycles allows for the wavelet convolution method to provide a better frequency resolution at lower frequencies and a better temporal resolution at higher frequencies. ERSP data were computed in decibels relative to baseline for all electrodes separately. The time-frequency data of all trials were averaged for each participant and condition separately, resulting in 2 average time-frequency maps for each electrode.

From these maps, the mean power was extracted from the $\mathrm{Cz}$ electrode in the following regions of interest (time $\times$ frequency) based on previous studies reviewed in [56]: from 2 to $10 \mathrm{~Hz}$ between 150 and $400 \mathrm{~ms}$, from 8 to $29 \mathrm{~Hz}$ between 300 and $1000 \mathrm{~ms}$, from 30 to $60 \mathrm{~Hz}$ between 100 and $350 \mathrm{~ms}$, and from 61 to $100 \mathrm{~Hz}$ between 150 and $350 \mathrm{~ms}$. The gamma-band was separated as low and high gamma based on previous studies $[3,15]$. The ERSP values for each time-frequency point included in the regions of interest were extracted from each subject. A mean ERSP value was then obtained for each participant and regions of interest by selecting and averaging the values with the $20 \%$ highest or lowest amplitude (for power increase or decrease relative to baseline) [32, 33, $45,48]$. The mean power calculations were performed on data from the same 60 participants used for $A \delta$-LEP analysis.

\section{Statistical analysis}

Statistical analysis was conducted using Statistica v13.1 (Kivuto Solutions Inc., Ottawa, ON, Canada). All results are expressed as mean $\pm \mathrm{SD}$. SD values were corrected to remove between-subject variability [14] and statistical threshold was set at $\mathrm{p}<0.05$. Data distribution was assessed for normality with the Kolmogorov-Smirnov test and homogeneity of variance was assessed using Levene's test. Since both tests indicated that the assumptions for using analysis of variance (ANOVA) were met, pain intensity, $\mathrm{A} \delta$ - $\mathrm{N} 2$ and $\mathrm{A} \delta$-P2 peak amplitude and latency, as well as ERSP values were analyzed using mixed ANOVA with one between-subject factor (groups, 4 levels) and one within-subject factor (time, 2 levels). Planned contrasts were used to decompose significant effects. For C-N2 and C-P2 analyses, the number of participants that could be included was limited and data was not normally distributed. Thus, groups were compared 
Table 1 Pain ratings for the four experimental groups $($ mean \pm SD)

\begin{tabular}{lllll}
\hline & No intervention & Placebo intervention & SM at T4 & SM at T8 \\
\hline PRE & $27.8 \pm 4.6$ & $27.5 \pm 3.9$ & $25.2 \pm 2.8$ & $23.1 \pm 2.6$ \\
POST & $28.1 \pm 4.6$ & $27.0 \pm 3.9$ & $19.8 \pm 2.8$ & $19.2 \pm 2.6$ \\
\hline
\end{tabular}

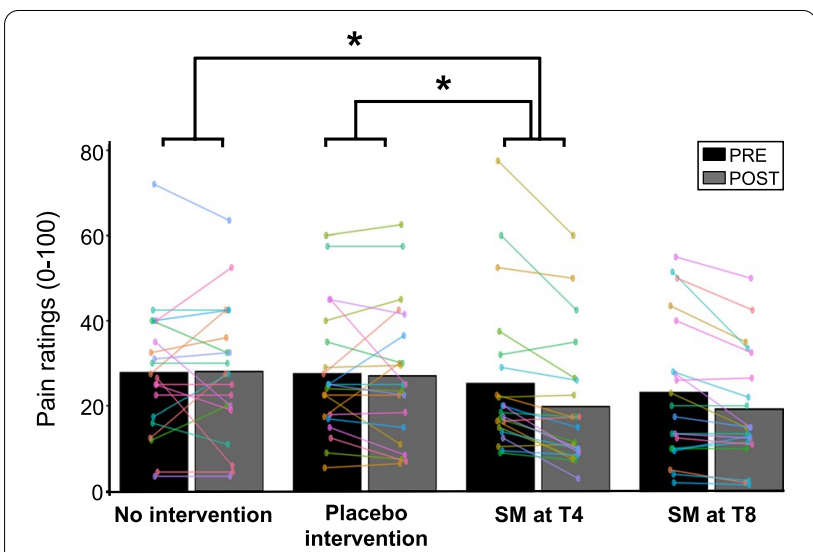

Fig. 3 Pain modulation. Mean pain ratings before and after the intervention for the four groups. SM at T4 (segmental) produced pain inhibition compared with no intervention or the placebo intervention. SM at T8 produced similar effects but they were not statistically significant compared with no intervention or the placebo intervention. Data from each participant are represented by linked colored dots and the mean of these data points for each condition are represented by black and grey bars. ${ }^{*} P<0.05$

on $\mathrm{C}-\mathrm{N} 2$ and C-P2 amplitude modulation using the Kruskal-Wallis $\mathrm{H}$ tests. Effect sizes are reported based on partial eta-squared $\left(\eta_{p}^{2}\right)$.

\section{Results}

\section{Pain intensity}

The mean stimulus intensity for each group was $5.0 \pm 0.4$ for no intervention, $4.5 \pm 1.3$ for the placebo intervention, $4.8 \pm 0.7$ for SM-T4 and $4.7 \pm 0.6$ for SM-T8. No significant difference was observed between groups $\left(\mathrm{F}_{3,78}=1.4, p=0.24 ; \eta_{p}^{2}=0.05\right)$. Pain ratings are reported in Table 1 and presented in Fig. 3. Pain intensity was significantly different between groups over time (interaction: $\left.F_{3,78}=2.97, p=0.037, \eta_{p}^{2}=0.10\right)$. Planned contrasts revealed that pain intensity was significantly decreased by $\mathrm{SM}$ at $\mathrm{T} 4$ compared with no intervention $(p=0.013)$ and the placebo intervention $(p=0.028)$. In contrast, SM at T8 did not modulate pain significantly compared with no intervention $(p=0.07)$ and the placebo intervention $(p=0.13)$. Moreover, the placebo intervention did not modulate pain compared with no intervention $(p=0.74)$. This indicates that pain inhibition by SM at T4 (homosegmental to pain stimulation) was greater than changes produced by non-specific temporal effects (no intervention) and placebo effects (light mechanical stimulation), while SM at T8 (heterosegmental to pain stimulation) did not produce significant effects. The same results were obtained with the 60 participants included in the EEG analyses (see below).

\section{Expectations of pain relief do not predict pain inhibition} Expectations of pain relief were $15.1 \pm 27.8$ for the placebo intervention, $20.2 \pm 21.1$ for $\mathrm{SM}$ at $\mathrm{T} 4$ and $13.8 \pm 16.7$ for $\mathrm{SM}$ at $\mathrm{T} 8$, with no significant difference between groups $\left(F_{2,59}=0.5, p=0.6, \eta_{p}^{2}=0.02\right)$. To examine whether greater expectation of pain relief predicted pain inhibition, simple regression analyses were performed. Pain inhibition was not predicted by expectations for the placebo $\left(R^{2}=0.08, p=0.22\right), \mathrm{SM}$ at $\mathrm{T} 4$ $\left(R^{2}=0.16, p=0.07\right)$ or SM at T8 $\left(R^{2}=0.005, p=0.76\right)$.

\section{Laser-evoked potentials}

Average waveforms and topographic maps for the N2 and P2 components are presented in Figs. 4, 5, 6 and 7. As expected, both components show a central scalp distribution and are maximal at the vertex.

\section{A $\delta$-N2 and $A \delta-P 2$ peak amplitude and latency}

A $\delta$-N2 peak amplitudes and latencies are presented in Tables 2 and 3 and Fig. 5. A $\delta$-N2 peak amplitude decreased over time (main effect: $\mathrm{F}_{1,56}=24.49, \mathrm{p}<0.001$, $\eta_{p}^{2}=0.30$ ), but this effect was not significantly different between groups (interaction: $\mathrm{F}_{3,56}=0.45, \mathrm{p}=0.72$, $\left.\eta_{p}^{2}=0.02\right)$. A $\delta$-N2 peak latency was not significantly different between groups (main effect: $\mathrm{F}_{3,56}=0.78, \mathrm{p}=0.51$, $\eta_{p}^{2}=0.04$ ) or between groups over time (interaction: $\left.\mathrm{F}_{3,56}=0.98, \mathrm{p}=0.41, \eta_{p}^{2}=0.05\right)$.

A $\delta$-P2 peak amplitudes and latencies are presented in Tables 2 and 3 and Fig. 6. A $\delta$-P2 peak amplitude decreased over time (main effect: $\mathrm{F}_{1,56}=34.1, \mathrm{p}<0.001$, $\eta_{p}^{2}=0.38$ ), but this effect was not significantly different between groups (interaction: $\mathrm{F}_{3,56}=1.52, \mathrm{p}=0.22$, $\left.\eta_{p}^{2}=0.08\right)$. A $\delta$-P2 peak latency was not significantly different between groups (main effect: $\mathrm{F}_{3,56}=1.0, \mathrm{p}=0.40$, $\eta_{p}^{2}=0.05$ ) or between groups over time (interaction: $\left.\mathrm{F}_{3,56}=0.36, \mathrm{p}=0.78, \eta_{p}^{2}=0.02\right)$.

\section{C-N2 and C-P2 peak amplitude and latency}

C-P2 peak amplitudes and latencies are reported in Tables 2 and 3 and presented in Fig. 8. Due to the low number of participants included in these analyses and low statistical power, no conclusion could be reached, and the results are presented as pilot data only. The Kruskal-Wallis $\mathrm{H}$ test revealed no significant difference between groups for the $\mathrm{C}-\mathrm{N} 2$ peak $(\mathrm{H}(3)=7.3, \mathrm{p}=0.062)$, the C-P2 peak $(\mathrm{H}(3)=3.0, \mathrm{p}=0.40)$, the $\mathrm{C}-\mathrm{N} 2$ latency 

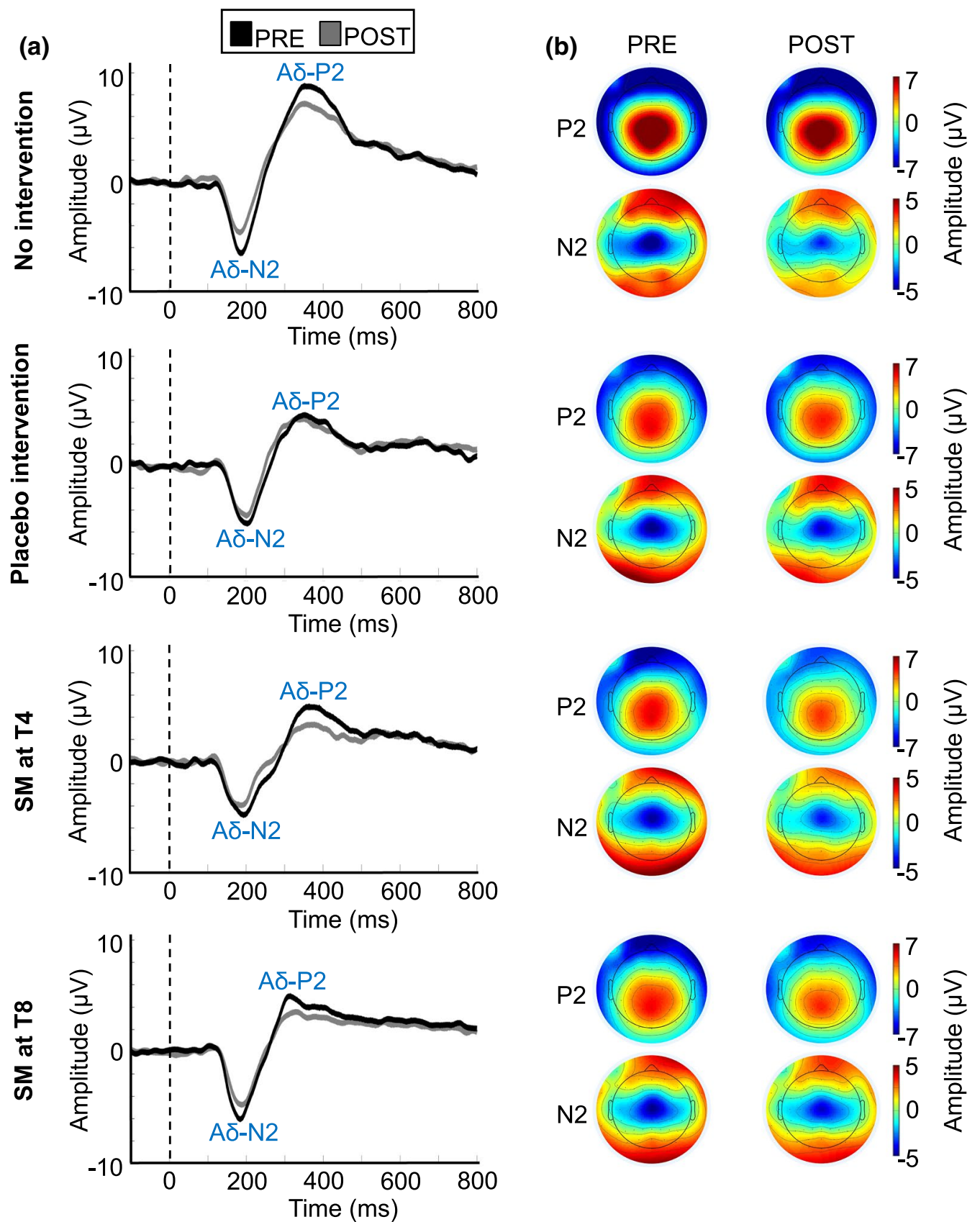

Fig. 4 A $\delta$-fiber laser-evoked potentials. a Average waveforms time-locked to the onset of laser stimulation for the N2 and P2 at Cz with a nose

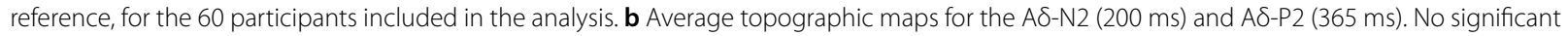
effect was observed between groups

$(\mathrm{H}(3)=1.7, \mathrm{p}=0.63)$, and C-P2 latency $(\mathrm{H}(3)=1.9$, $\mathrm{p}=0.59)$.

\section{Event-related spectral perturbations}

Averaged ERSPs and regions of interest are presented in Fig. 9.

\section{$2-10 \mathrm{~Hz}$}

The mean power in the $2-10 \mathrm{~Hz}$ region of interest is presented in Table 4 and Fig. 10a. It decreased over time (main effect: $F_{1,56}=52.7, p<0.001, \eta_{p}^{2}=0.48$ ), but this effect was not significantly different between groups (interaction: $F_{3,56}=0.68, p=0.57, \eta_{p}^{2}=0.04$ ). 


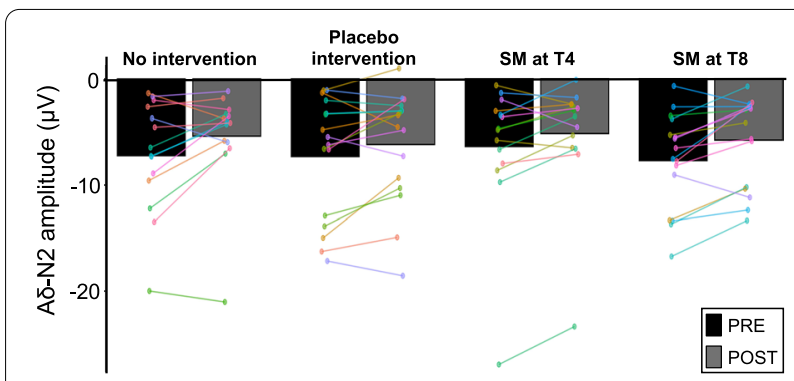

Fig. 5 A $\delta-P 2$ amplitude. Mean P2 peak amplitude. No significant effect was observed between groups. Data from each participant are represented by linked colored dots and the mean of these data points for each condition are represented by black and grey bars

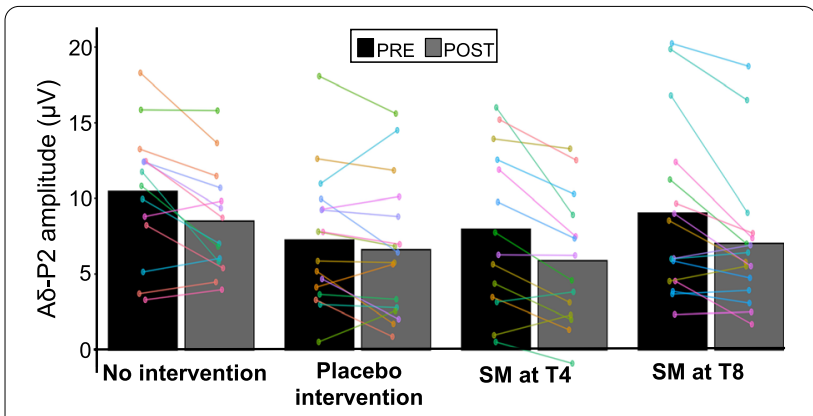

Fig. 6 A $\delta-N 2$ amplitude. Mean A $\delta-N 2$ peak amplitude. No significant effect was observed between groups. Data from each participant are represented by linked colored dots and the mean of these data points for each condition are represented by black and grey bars

\section{8-29 Hz}

The mean power in the $8-29 \mathrm{~Hz}$ region of interest is presented in Table 4 and Fig. 10b. It increased over time (main effect: $F_{1,56}=13.5, p<0.001, \eta_{p}^{2}=0.19$ ), but this effect was not significantly different between groups (interaction: $\mathrm{F}_{3,56}=0.45, \mathrm{p}=0.72, \eta_{p}^{2}=0.02$ ).

\section{$30-60 \mathrm{~Hz}$}

The mean power in the $30-60 \mathrm{~Hz}$ region of interest is presented in Table 4 and Fig. 10c. It was not significantly different over time (main effect: $F_{1,56}=0.05$, $p=0.82, \eta_{p}^{2}=0.0009$ ) or between groups over time (interaction: $F_{3,56}=0.55, p=0.65, \eta_{p}^{2}=0.03$ ).

\section{$61-100 \mathrm{~Hz}$}

The mean power in the $61-100 \mathrm{~Hz}$ region of interest is presented in Table 4 and Fig. 10d. It was not significantly different over time (main effect: $F_{1,56}=1.70$, $p=0.20, \eta_{p}^{2}=0.03$ ) or between groups over time (interaction: $F_{3,56}=2.62, p=0.06, \eta_{p}^{2}=0.12$ ).

\section{Discussion}

The aim of this study was to determine the neurophysiological mechanisms of SM-induced hypoalgesia by examining changes in nociceptive brain activity evoked by laser stimulation. The expected decrease in nociceptive brain activity would reflect a downstream inhibition of spinal nociceptive transmission considering that SMinduced hypoalgesia is presumed to be caused by spinal processes $[7,8,25,50]$. The present results indicate that SM applied segmentally, but not heterosegmentally to the laser stimulus reduces laser-evoked pain compared with a placebo intervention and non-specific temporal effects. Brain activity related to A $\delta$ fiber activation were not significantly modulated by the intervention, confirming our hypothesis that SM-induced hypoalgesia is produced by inhibition of nociceptive processes that are independent of $A \delta$ fibers. However, brain activity related to $\mathrm{C}$-fibers could not be measured reliably so it remains to be clarified whether SM-induced hypoalgesia relies on the inhibition of activity related to $\mathrm{C}$-fibers.

Pain inhibition by SM was examined in several studies using mechanical, electrical, chemical (capsaicin) or thermal stimuli [42]. Generally, these studies indicate that SM produces hypoalgesia. A series of studies also indicate that pain inhibition by SM relies more specifically on processes related to $C$-fiber activity $[6,7,9,25,50]$. This conclusion was based on the lack of SM-induced hypoalgesia when pain was evoked by a single contact heat stimulus, while TSP produced by a repetition of the same stimulus at a frequency known to produce wind-up was decreased. In most of these studies, painful stimuli were applied on the leg, in a lumbar dermatome related to vertebral segments where SM was applied and not on the back, to allow measuring specific effects on A $\delta$ and C-fibers [25]. A subsequent study confirmed that inhibition of TSP by SM could also be observed when painful stimuli are applied on the back, at the site of SM [60]. In that study, pain evoked by a single electric pulse was not inhibited while pain evoked by a repeated application of the same stimulus (TSP) was decreased. In the present study, the lack of inhibition of A $\delta$-fibers' activity was shown experimentally with measures of brain activity. This is the first study to investigate the neurophysiological effects of SM using laser stimulation, which allows the activation of nociceptive afferents selectively and the assessment of nociceptive brain activity $[11,55]$. In addition to LEPs, ERSPs were examined. The lack of effect of SM on painrelated ERSPs is consistent with some of the findings in a previous study, in which the effects of SM on central processing of tonic pain were examined [47]. Indeed, no significant change was observed in delta, theta, alpha and beta frequency bands after one session of multiple SM in healthy volunteers. Furthermore, since laser stimuli 


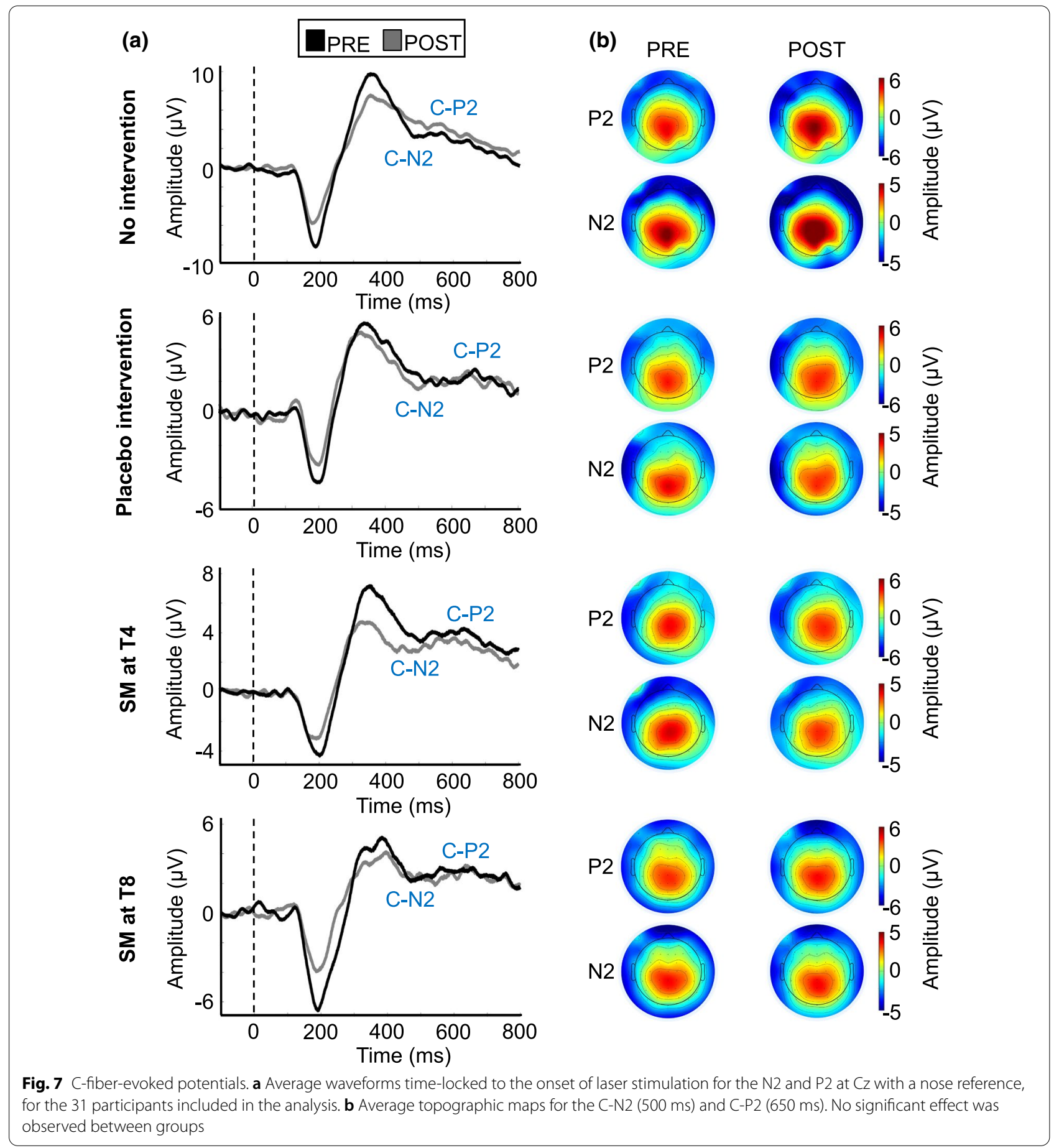

activate $\mathrm{A} \delta$ and $\mathrm{C}$-fibers, the significant pain inhibition observed in the SM-T4 group is likely caused by inhibition of $\mathrm{C}$-fiber-related processes. However, this could not be confirmed because laser-evoked C-fiber activity could not be measured reliably in most participants, although the experimental protocol was adapted to allow detecting such activity [31]. It is possible that the number of stimuli was not sufficient to obtain optimal signal to noise ratio, but 40 stimuli per condition was a compromise to avoid tissue damage or non-specific temporal effects. It is also possible that the different innervation of back and hand skin by nociceptors makes C-fiber LEP measurement more difficult in the back. However, a recent study indicates that the spatial acuity for pain is comparable 
Table 2 N2 and P2 peak amplitude $(\mu \mathrm{V})$ for the four experimental groups (mean \pm SD)

\begin{tabular}{|c|c|c|c|c|c|c|}
\hline & & & No intervention & Placebo intervention & SM at T4 & SM at T8 \\
\hline \multirow[t]{4}{*}{$A \delta$} & N2 & PRE & $-7.2 \pm 1.5$ & $-7.3 \pm 1.2$ & $-6.4 \pm 1.0$ & $-7.7 \pm 1.1$ \\
\hline & & POST & $-5.4 \pm 1.5$ & $-6.2 \pm 1.2$ & $-5.1 \pm 1.0$ & $-5.7 \pm 1.1$ \\
\hline & P2 & PRE & $10.5 \pm 1.1$ & $7.3 \pm 1.0$ & $8.0 \pm 1.1$ & $9.0 \pm 1.2$ \\
\hline & & POST & $8.5 \pm 1.1$ & $6.6 \pm 1.0$ & $5.9 \pm 1.1$ & $7.0 \pm 1.2$ \\
\hline \multirow[t]{4}{*}{ C } & N2 & PRE & $2.2 \pm 0.71$ & $0.49 \pm 0.46$ & $1.8 \pm 0.78$ & $0.42 \pm 1.3$ \\
\hline & & POST & $3.5 \pm 0.71$ & $0.25 \pm 0.46$ & $1.0 \pm 0.78$ & $0.57 \pm 1.3$ \\
\hline & P2 & PRE & $4.4 \pm 0.82$ & $3.8 \pm 0.42$ & $5.4 \pm 0.62$ & $3.8 \pm 1.2$ \\
\hline & & POST & $4.9 \pm 0.82$ & $3.5 \pm 0.42$ & $4.8 \pm 0.62$ & $4.2 \pm 1.2$ \\
\hline
\end{tabular}

Table 3 N2 and P2 peak latency (ms) for the four experimental groups (mean \pm SD)

\begin{tabular}{|c|c|c|c|c|c|c|}
\hline & & & No intervention & Placebo intervention & $\mathrm{SM}$ at T4 & $\mathrm{SM}$ at $\mathrm{T} 8$ \\
\hline \multirow[t]{4}{*}{$A \delta$} & N2 & PRE & $201.9 \pm 8.5$ & $220.8 \pm 9.1$ & $217.0 \pm 7.5$ & $224.1 \pm 15.3$ \\
\hline & & POST & $195.6 \pm 8.5$ & $208.4 \pm 9.1$ & $214.9 \pm 7.5$ & $223.6 \pm 15.3$ \\
\hline & $\mathrm{P} 2$ & PRE & $377.9 \pm 21.4$ & $398.3 \pm 41.7$ & $407.4 \pm 35.8$ & $398.8 \pm 16.2$ \\
\hline & & POST & $361.0 \pm 21.4$ & $393.0 \pm 41.7$ & $411.5 \pm 35.8$ & $401.8 \pm 16.2$ \\
\hline \multirow[t]{4}{*}{ C } & N2 & PRE & $543.7 \pm 13.3$ & $513.8 \pm 11.0$ & $476.6 \pm 16.5$ & $483.0 \pm 9.4$ \\
\hline & & POST & $544.7 \pm 13.3$ & $521.2 \pm 22.0$ & $465.7 \pm 16.5$ & $489.2 \pm 9.4$ \\
\hline & P2 & PRE & $603.5 \pm 17.0$ & $601.7 \pm 16.5$ & $577.8 \pm 21.0$ & $596.4 \pm 8.1$ \\
\hline & & POST & $594.9 \pm 17.0$ & $609.9 \pm 16.5$ & $575.8 \pm 21.0$ & $586.6 \pm 8.1$ \\
\hline
\end{tabular}

between these two regions [41] so it is unlikely that sparser innervation in the back explains the lack of measurable C-fiber LEP in the present study. Nevertheless, the shorter distance between the skin of $\mathrm{T} 4$ region and the brain may not allow $\mathrm{A} \delta$ - and $\mathrm{C}$-fiber responses to be distinguished as clearly as for the hand. Therefore, it remains to be clarified whether $\mathrm{C}$-fiber activity is inhibited by SM and whether this contributes to pain inhibition or whether SM has a specific effect on pain amplification processes such as wind-up and the resulting TSP. Future studies are needed to optimize stimulation protocols and experimental designs to examine the modulation of nociceptive brain activity by SM with laser stimulation in the back. Another issue to address is the non-specific attenuation of laser-evoked brain responses, including $\mathrm{A} \delta$ - and $\mathrm{C}$-fiber responses. The amplitude of some nociceptive responses measures in the present study attenuated over time. It is known that stimulus saliency and their evoked neurophysiological responses decrease as the number of stimuli of constant intensity increases [33, 61]. However, it may be possible to limit this attenuation by delivering the laser stimuli among non-painful stimuli, making laser stimuli unpredictable, novel and more salient [17, 18, 38].

In the present study, we also examined whether nociceptive activity and laser-evoked pain may be modulated by a light mechanical stimulus (the placebo intervention).
The lack of pain inhibition by this placebo intervention suggests that SM produced specific effects and that SMinduced hypoalgesia relies at least in part on the activation of deep high-threshold mechanoreceptors. This is congruent with current knowledge on SM hypoalgesic mechanisms. A body of knowledge from animal and human studies indicates that spinal manipulation transiently increases the discharge of type Ia, Ib and II afferents in paraspinal tissues $[53,54]$. These afferents and the nociceptive afferents project to the dorsal horn of the spinal cord where they may interact [53]. Although this remains to be clarified, the inhibition of TSP by SM may represent a perceptual correlate of this spinal interaction $[25,60]$. In addition, the present results corroborate findings from a previous study in which no significant hypoalgesia occurred following the application of a light mechanical stimulus [60]. Yet, Penza et al. reported a similar inhibition of TSP when comparing low-velocity, low-amplitude spinal mobilization to SMT [50]. Nevertheless, the amount of force applied during this mobilization was not measured, so it may have been sufficient to activate deep high-threshold mechanoreceptors.

Previous studies have examined how psychological factors may contribute to SM-induced hypoalgesia $[5,8,10]$. In the present study, to limit a potential bias induced by knowledge on SM, chiropractors or chiropractic students 


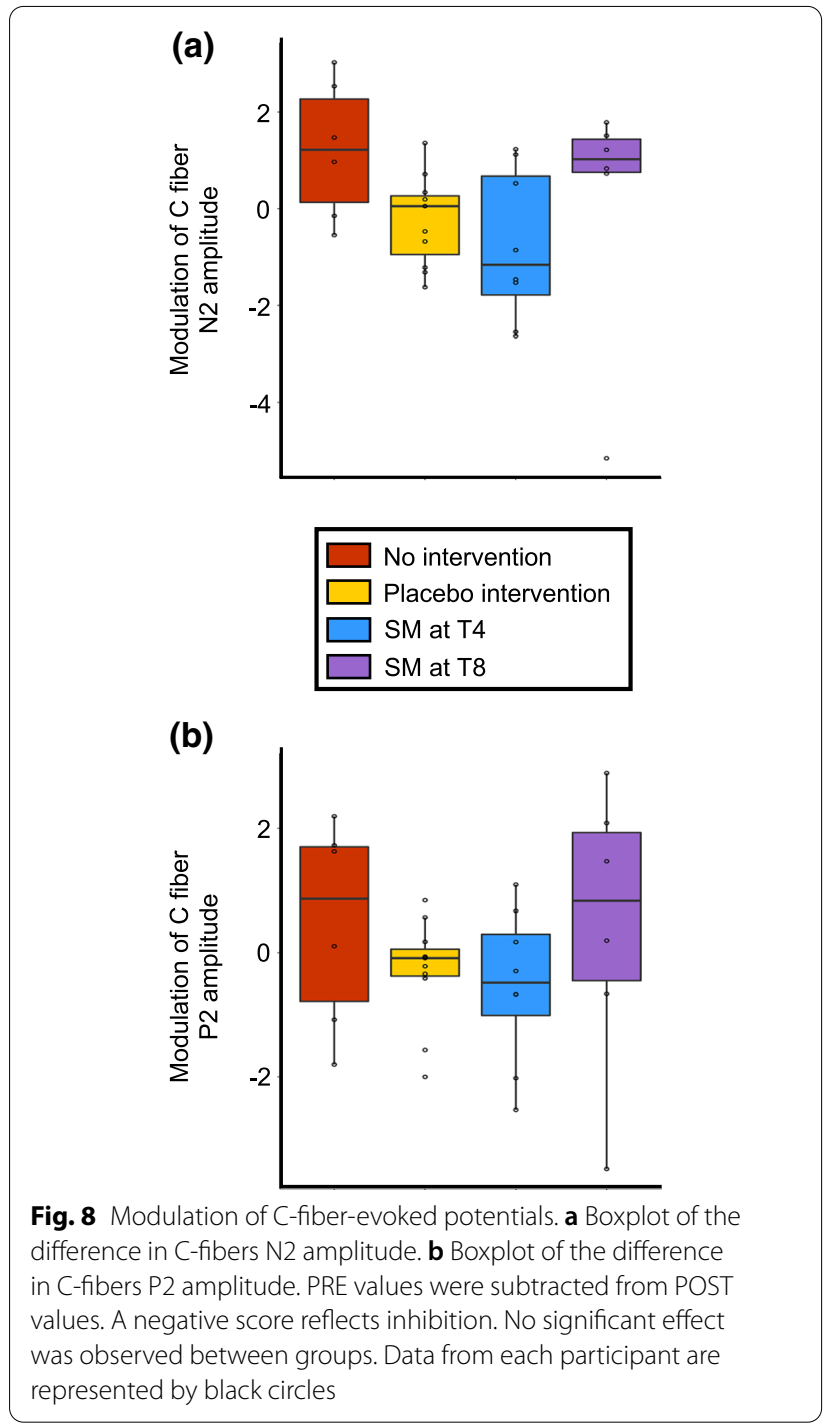

were not included in the recruited sample. Indeed, knowledge on SM and their pain-relieving effects may be associated with expectation of pain relief, which may affect the results. In addition, expectations of pain relief were measured, and this confirmed that expectations were not significantly different between groups. Moreover, expectations did not predict pain inhibition. Thus, it is unlikely that pain inhibition by SM was caused by expectations in the present study.

In the present experimental study, we included healthy volunteers with no spine disorder, no spine pain, and no clinical indication for SM. Several studies have demonstrated the hypoalgesic effects of SM performed on arbitrarily selected spinal segments in healthy volunteers $[6,9,25,50,60]$. This indicates that SM produces hypoalgesic effects even in the absence of
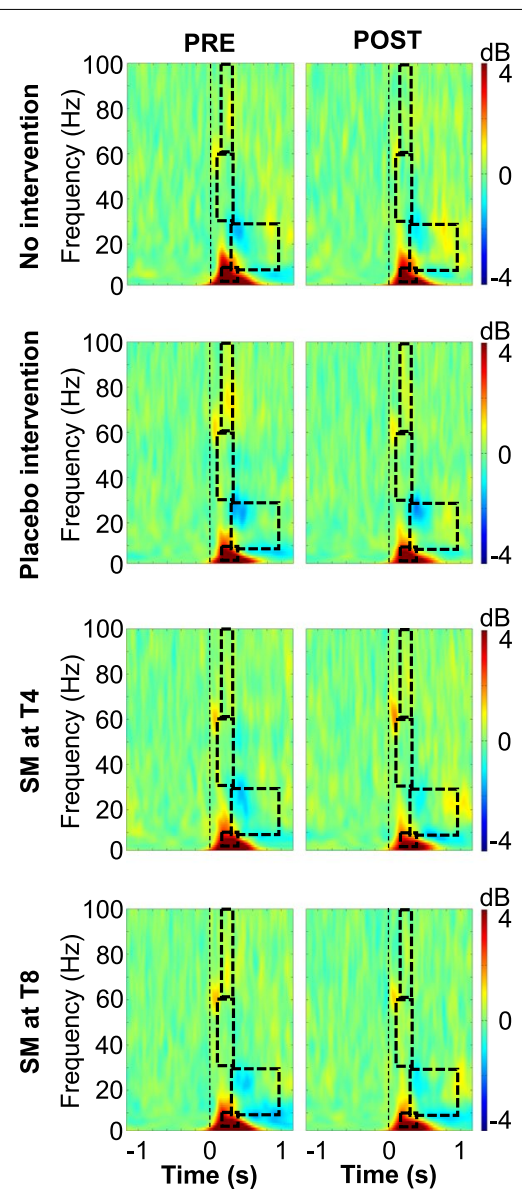

Fig. 9 Time-frequency analyses. Average time-frequency maps, time-locked to the onset of laser stimulation. Units are in decibels relative to baseline (-700-- $200 \mathrm{~ms})$. Regions of interest are highlighted by the dashed rectangles. No significant effect was observed between groups

a clinical indication. Whether the hypoalgesic effects measured in healthy volunteers are different compared with patients with back pain or spine disorders remains to be clarified [65].

Regarding heterosegmental effects, inhibition of laserevoked pain was not significant when SM was performed at T8 (SM-T8 group). However, a similar trend as for the segmental SM (SM-T4 group) was observed so we cannot exclude that weaker, but significant effects may be observed with larger samples. Also, T4 and T8 vertebrae are relatively close to each other so this result suggests that the hypoalgesic effects may show a gradient, with the amplitude of the effects decreasing as a function of the distance from the manipulated segment. Previous studies reported a lack of pain inhibition by heterosegmental SM $[7,25,50]$, but in these studies, the heterosegmental SM was in a different spinal region. Besides, although the SM 
Table 4 ERSP power (dB) for the four experimental groups (mean $\pm S D$ )

\begin{tabular}{llccrr}
\hline & & No intervention & Placebo intervention & SM at T4 & SM at T8 \\
\hline $2-10 \mathrm{~Hz}$ & PRE & $8.4 \pm 0.8$ & $7.2 \pm 0.7$ & $7.8 \pm 0.7$ & $6.1 \pm 0.7$ \\
& POST & $7.0 \pm 0.8$ & $6.1 \pm 0.7$ & $-2.0 \pm 0.4$ & $-2.3 \pm 0.9$ \\
$8-29 \mathrm{~Hz}$ & PRE & $-1.8 \pm 0.3$ & $-2.1 \pm 0.3$ & $-1.7 \pm 0.4$ & $-1.8 \pm 0.4$ \\
& POST & $-1.4 \pm 0.3$ & $-1.9 \pm 0.3$ & $1.1 \pm 0.2$ & $1.1 \pm 0.3$ \\
$30-60 \mathrm{~Hz}$ & PRE & $0.9 \pm 0.3$ & $1.0 \pm 0.3$ & $0.9 \pm 0.2$ & $1.1 \pm 0.3$ \\
& POST & $1.0 \pm 0.3$ & $1.1 \pm 0.3$ & $1.4 \pm 0.4$ & $1.6 \pm 0.2$ \\
$61-100 \mathrm{~Hz}$ & PRE & $1.4 \pm 0.4$ & $1.8 \pm 0.2$ & $1.6 \pm 0.4$ & $1.3 \pm 0.2$ \\
& POST & $1.4 \pm 0.4$ & $1.6 \pm 0.2$ & & \\
\hline
\end{tabular}

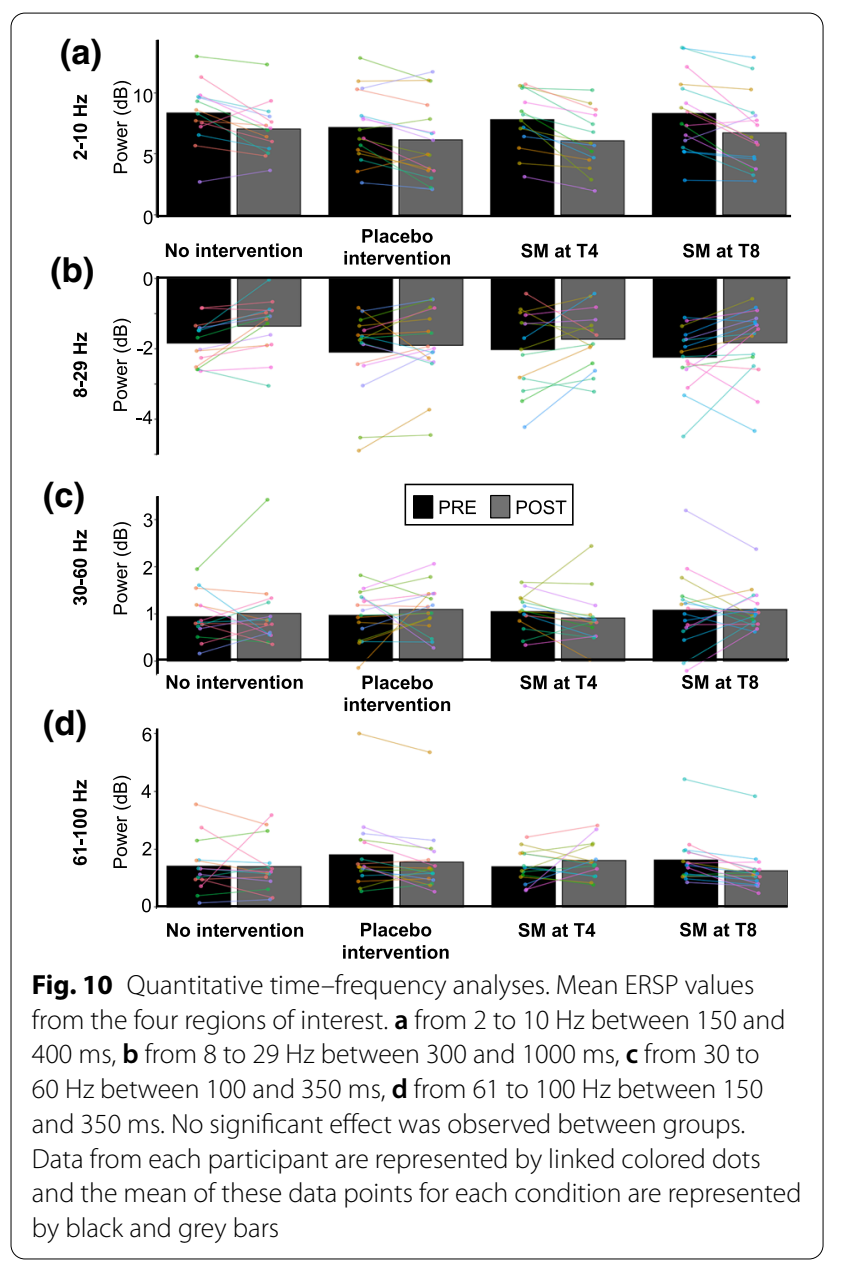

technique used in the present study aims at specific joints (T4-T5), several joints are mobilized [23, 49].

\section{Conclusion}

In summary, SM can reduce pain evoked by laser stimulation in the back and this hypoalgesic effect does not involve nociceptive processing related to A $\delta$-fiber activation. It remains to be confirmed whether $\mathrm{C}$-fiber activity is inhibited by SM and whether this contributes to its pain-relieving effects.

\section{Abbreviations}

EEG: Electroencephalography; EOG: Electrooculography; ERSP: Event-related spectral perturbations; FIR: Finite impulse response; LBP: Low back pain; LEP: Laser-evoked potential; NdYAP: Neodymium-doped yttrium aluminum perovskite laser; SM-T4: Spinal manipulation at T4 (segmental to laser-evoked pain); SM-T8: Spinal manipulation at T8 (heterosegmental to laser-evoked pain); SM: Spinal manipulation; SMT: Spinal manipulative therapy; TSP: Temporal summation of pain.

\section{Acknowledgements}

Not applicable

\section{Authors' contributions}

Each author has contributed significantly to this work and has read and approved the final version of the manuscript. BP contributed to data collection, analysis and interpretation and wrote the preliminary version of the manuscript. SN contributed to data collection and analyses. CG-M and JO contributed to data collection. MP contributed to study design, data collection, analyses, and interpretation, wrote the final version of the manuscript and obtained funding for the study. All authors read and approved the final manuscript.

\section{Funding}

This project was funded by the Fondation Chiropratique du Québec, the Natural Science and Engineering Research Council of Canada (\#06659) and the Canadian Foundation for Innovation (\#33731). The contribution of Benjamin Provencher was supported by the Canadian Institutes of Health Research (CIHR) and the Fonds de Recherche du Québec-Santé (FRQS). The contribution of Carlos Gevers Montoro was supported by the Fondation Chiropratique du Québec. The contribution of Mathieu Piché was supported by the Fonds de Recherche du Québec-Santé (FRQS).

\section{Availability of data and materials}

The dataset supporting the conclusions of this article is available from the corresponding author on reasonable request.

\section{Declarations}

Ethics approval and consent to participate

All experimental procedures conformed to the standards set by the latest revision of the Declaration of Helsinki and were approved by the Research Ethics Board of Université du Québec à Trois-Rivières. All participants gave written informed consent, acknowledging their right to withdraw from the experiment without prejudice, and received a compensation of $\$ 25$ for their travel expenses, time and commitment. 


\section{Consent for publication \\ Not applicable.}

\section{Competing interests}

The authors declare that they have no competing interests.

\begin{abstract}
Author details
'Department of Anatomy, Université du Québec à Trois-Rivières, 3351 boul. des Forges, C.P. 500, Trois-Rivières, QC G9A 5H7, Canada. ${ }^{2}$ CogNAC Research Group, Université du Québec à Trois-Rivières, Trois-Rivières, QC G9A 5H7, Canada. ${ }^{3}$ Madrid College of Chiropractic, Madrid, Spain. ${ }^{4}$ Department of Chiropractic, Université du Québec à Trois-Rivières, Trois-Rivières, QC G9A 5H7, Canada.
\end{abstract}

\section{Received: 24 February 2021 Accepted: 15 June 2021}

Published online: 24 June 2021

\section{References}

1. Arendt-Nielsen $L$ (2015) Central sensitization in humans: assessment and pharmacology. Handb Exp Pharmacol 227:79-102. https://doi.org/10. 1007/978-3-662-46450-2_5

2. Aspinall SL, Jacques A, Leboeuf-Yde C, Etherington SJ, Walker BF (2019) No difference in pressure pain threshold and temporal summation after lumbar spinal manipulation compared to sham: a randomised controlled trial in adults with low back pain. Musculoskelet Sci Pract 43:18-25. https://doi.org/10.1016/j.msksp.2019.05.011

3. Babiloni C, Babiloni F, Carducci F, Cincotti F, Rosciarelli F, Arendt-Nielsen L, Chen AC, Rossini PM (2002) Human brain oscillatory activity phase-locked to painful electrical stimulations: a multi-channel EEG study. Hum Brain Mapp 15(2):112-123. https://doi.org/10.1002/hbm.10013

4. Bialosky JE, Beneciuk JM, Bishop MD, Coronado RA, Penza CW, Simon CB, George SZ (2018) Unraveling the mechanisms of manual therapy: modeling an approach. J Orthop Sports Phys Ther 48(1):8-18. https://doi. org/10.2519/jospt.2018.7476

5. Bialosky JE, Bishop MD, Penza CW (2017) Placebo mechanisms of manual therapy: a sheep in wolf's clothing? J Orthop Sports Phys Ther 47(5):301-304. https://doi.org/10.2519/jospt.2017.0604

6. Bialosky JE, Bishop MD, Robinson ME, Barabas JA, George SZ (2008) The influence of expectation on spinal manipulation induced hypoalgesia: an experimental study in normal subjects. BMC Musculoskelet Disord 9:19. https://doi.org/10.1186/1471-2474-9-19

7. Bialosky JE, Bishop MD, Robinson ME, Zeppieri G Jr, George SZ (2009) Spinal manipulative therapy has an immediate effect on thermal pain sensitivity in people with low back pain: a randomized controlled trial. Phys Ther 89(12):1292-1303. https://doi.org/10.2522/ptj.20090058

8. Bialosky JE, George SZ, Horn ME, Price DD, Staud R, Robinson ME (2014) Spinal manipulative therapy-specific changes in pain sensitivity in individuals with low back pain (NCT01168999). J Pain 15(2):136-148. https:// doi.org/10.1016/j.jpain.2013.10.005

9. Bishop MD, Beneciuk JM, George SZ (2011) Immediate reduction in temporal sensory summation after thoracic spinal manipulation. Spine J 11(5):440-446. https://doi.org/10.1016/j.spinee.2011.03.001

10. Bishop MD, Bialosky JE, Penza CW, Beneciuk JM, Alappattu MJ (2017) The influence of clinical equipoise and patient preferences on outcomes of conservative manual interventions for spinal pain: an experimental study. J Pain Res 10:965-972. https://doi.org/10.2147/JPR.S130931

11. Bromm B, Jahnke MT, Treede RD (1984) Responses of human cutaneous afferents to CO2 laser stimuli causing pain. Exp Brain Res 55(1):158-166. https://doi.org/10.1007/BF00240510

12. Bussières $A E$, Stewart $G, A l$-Zoubi F, Decina P, Descarreaux M, Haskett D, Hincapié C, Pagé I, Passmore S, Srbely J, Stupar M (2018) Spinal manipulative therapy and other conservative treatments for low back pain: a guideline from the canadian chiropractic guideline initiative. J Manipulative Physiol Ther 41(4):265-293. https://doi.org/10.1016/j.jmpt.2017.12. 004

13. Côté $P$, Wong JJ, Sutton D, Shearer HM, Mior S, Randhawa K, Ameis A, Carroll LJ, Nordin M, Yu H, Lindsay GM (2016) Management of neck pain and associated disorders: a clinical practice guideline from the Ontario
Protocol for Traffic Injury Management (OPTIMa) Collaboration. Eur Spine J 25(7):2000-2022. https://doi.org/10.1007/s00586-016-4467-7

14. Cousineau D (2005) Confidence intervals in within-subject designs: a simpler solution to Loftus and Masson's method. TQMP 1(1):42-45. https:// doi.org/10.20982/tqmp.01.1.p042

15. Croft RJ, Williams JD, Haenschel C, Gruzelier JH (2002) Pain perception, hypnosis and $40 \mathrm{~Hz}$ oscillations. Int J Psychophysiol 46(2):101-108. https://doi.org/10.1016/s0167-8760(02)00118-6

16. Cruccu G, lannetti GD, Agostino R, Romaniello A, Truini A, Manfredi M (2000) Conduction velocity of the human spinothalamic tract as assessed by laser evoked potentials. NeuroReport 11(13):3029-3032. https://doi. org/10.1097/00001756-200009110-00040

17. Deldar Z, Rustamov N, Blanchette I, Piche M (2019) Improving working memory and pain inhibition in older persons using transcranial direct current stimulation. Neurosci Res 148:19-27. https://doi.org/10.1016/j. neures.2018.12.007

18. Deldar Z, Rustamov N, Bois S, Blanchette I, Piche M (2018) Enhancement of pain inhibition by working memory with anodal transcranial direct current stimulation of the left dorsolateral prefrontal cortex. J Physiol Sci 68(6):825-836. https://doi.org/10.1007/s12576-018-0598-4

19. Delorme A, Makeig S (2004) EEGLAB: an open source toolbox for analysis of single-trial EEG dynamics including independent component analysis. J Neurosci Methods 134:9-21. https://doi.org/10.1016/j.jneumeth.2003. 10.009

20. Domnick C, Hauck M, Casey KL, Engel AK, Lorenz J (2009) C-fiber-related EEG-oscillations induced by laser radiant heat stimulation of capsaicintreated skin. J Pain Res 2:49-56. https://doi.org/10.2147/jpr.s4860

21. Foster NE, Anema JR, Cherkin D, Chou R, Cohen SP, Gross DP, Ferreira PH, Fritz JM, Koes BW, Peul W, Turner JA (2018) Prevention and treatment of low back pain: evidence, challenges, and promising directions. The Lancet 391(10137):2368-2383. https://doi.org/10.1016/S0140-6736(18) 30489-6

22. Franz M, Ritter A, Puta C, Nötzel D, Miltner WH, Weiss T (2014) Laser heat hyperalgesia is not a feature of non-specific chronic low back pain. Eur $\mathrm{J}$ Pain 18(10):1501-1508. https://doi.org/10.1002/ejp.535

23. Gál J, Herzog W, Kawchuk G, Conway PJ, Zhang YT (1997) Movements of vertebrae during manipulative thrusts to unembalmed human cadavers. J Manipulative Physiol Ther 20(1):30-40

24. Garcia-Larrea L, Frot M, Valeriani M (2003) Brain generators of laserevoked potentials: from dipoles to functional significance. Neurophysiol Clin 33(6):279-292. https://doi.org/10.1016/j.neucli.2003.10.008

25. George SZ, Bishop MD, Bialosky JE, Zeppieri G Jr, Robinson ME (2006) Immediate effects of spinal manipulation on thermal pain sensitivity: an experimental study. BMC Musculoskelet Disord 7:68-78. https://doi.org/ 10.1186/1471-2474-7-68

26. Goffaux P, Redmond WJ, Rainville P, Marchand S (2007) Descending analgesia-when the spine echoes what the brain expects. Pain 130(1-2):137143. https://doi.org/10.1016/j.pain.2006.11.011

27. Gyer G, Michael J, Inklebarger J, Tedla JS (2019) Spinal manipulation therapy: Is it all about the brain? A current review of the neurophysiological effects of manipulation. J Integr Med 17(5):328-337. https://doi.org/ 10.1016/j.joim.2019.05.004

28. Heid C, Mouraux A, Treede RD, Schuh-Hofer S, Rupp A, Baumgartner U (2020) Early gamma-oscillations as correlate of localized nociceptive processing in primary sensorimotor cortex. J Neurophysiol 123(5):17111726. https://doi.org/10.1152/jn.00444.2019

29. Herrero JF, Laird JM, Lopez-Garcia JA (2000) Wind-up of spinal cord neurones and pain sensation: much ado about something? Prog Neurobiol 61(2):169-203. https://doi.org/10.1016/s0301-0082(99)00051-9

30. Herzog W (2010) The biomechanics of spinal manipulation. J Bodyw Mov Ther 14(3):280-286. https://doi.org/10.1016/j.jbmt.2010.03.004

31. Hu L, Cai MM, Xiao P, Luo F, lannetti GD (2014) Human brain responses to concomitant stimulation of Adelta and C nociceptors. J Neurosci 34(34):11439-11451. https://doi.org/10.1523/JNEUROSCI.1355-14.2014

32. Hu L, Peng W, Valentini E, Zhang Z, Hu Y (2013) Functional features of nociceptive-induced suppression of alpha band electroencephalographic oscillations. J Pain 14(1):89-99. https://doi.org/10.1016/j.jpain. 2012.10.008 
33. lannetti GD, Hughes NP, Lee MC, Mouraux A (2008) Determinants of laser-evoked EEG responses: pain perception or stimulus saliency? J Neurophysiol 100(2):815-828. https://doi.org/10.1152/jn.00097.2008

34. Iannetti GD, Truini A, Romaniello A, Galeotti F, Rizzo C, Manfredi M, Cruccu $G$ (2003) Evidence of a specific spinal pathway for the sense of warmth in humans. J Neurophysiol 89(1):562-570. https://doi.org/10.1152/jn.00393. 2002

35. James SL, Abate D, Abate KH, Abay SM, Abbafati C, Abbasi N, Abbastabar H, Abd-Allah F, Abdela J, Abdelalim A, Abdollahpour I (2018) Global, regional, and national incidence, prevalence, and years lived with disability for 354 diseases and injuries for 195 countries and territories, 1990-2017: a systematic analysis for the Global Burden of Disease Study 2017. Lancet 392(10159):1789-1858. https://doi.org/10.1016/S01406736(18)32279-7

36. Jankovski A, Plaghki L, Mouraux A (2013) Reliable EEG responses to the selective activation of $\mathrm{C}$-fibre afferents using a temperature-controlled infrared laser stimulator in conjunction with an adaptive staircase algorithm. Pain 154(9):1578-1587. https://doi.org/10.1016/j.pain.2013.04.032

37. Kjaer P, Kongsted A, Hartvigsen J, Isenberg-Jørgensen A, SchiøttzChristensen B, Søborg B, Krog C, Møller CM, Halling CM, Lauridsen HH, Hansen IR (2017) National clinical guidelines for non-surgical treatment of patients with recent onset neck pain or cervical radiculopathy. Eur Spine J 26(9):2242-2257. https://doi.org/10.1007/s00586-017-5121-8

38. Legrain V, Crombez G, Plaghki L, Mouraux A (2013) Shielding cognition from nociception with working memory. Cortex 49(7):1922-1934. https:// doi.org/10.1016/j.cortex.2012.08.014

39. Luck SJ (2014) An introduction to the event-related potential technique, 2nd edn. The MIT Press, Cambridge

40. Madden VJ, Catley MJ, Grabherr L, Mazzola F, Shohag M, Moseley GL (2016) The effect of repeated laser stimuli to ink-marked skin on skin temperature-recommendations for a safe experimental protocol in humans. PeerJ 4:e1577. https://doi.org/10.7717/peerj.1577

41. Mancini F, Bauleo A, Cole J, Lui F, Porro CA, Haggard P, lannetti GD (2014) Whole-body mapping of spatial acuity for pain and touch. Ann Neurol 75(6):917-924. https://doi.org/10.1002/ana.24179

42. Millan M, Leboeuf-Yde C, Budgell B, Amorim MA (2012) The effect of spinal manipulative therapy on experimentally induced pain: a systematic literature review. Chiropr Man Therap 20(1):26. https://doi.org/10.1186/ 2045-709X-20-26

43. Moayedi M, Stefano G, Stubbs M, Djeugam BL, M, \& lannetti, G. (2016) Nociceptive-evoked potentials are sensitive to behaviorally relevant stimulus displacements in egocentric coordinates. ENeuro 3(3):e0151e0163. https://doi.org/10.1523/ENEURO.0151-15.2016

44. Mouraux A, Guérit JM, Plaghki L (2003) Non-phase locked electroencephalogram (EEG) responses to $\mathrm{CO} 2$ laser skin stimulations may reflect central interactions between $\mathrm{A} \partial$ - and C-fibre afferent volleys. Clin Neurophysiol 114(4):710-722. https://doi.org/10.1016/S1388-2457(03)00027-0

45. Mouraux A, lannetti GD (2008) Across-trial averaging of event-related EEG responses and beyond. Magn Reson Imaging 26(7):1041-1054. https:// doi.org/10.1016/j.mri.2008.01.011

46. Mouraux A, lannetti GD (2009) Nociceptive laser-evoked brain potentials do not reflect nociceptive-specific neural activity. J Neurophysiol 101(6):3258-3269. https://doi.org/10.1152/jn.91181.2008

47. Navid MS, Lelic D, Niazi IK, Holt K, Mark EB, Drewes AM, Haavik H (2019) The effects of chiropractic spinal manipulation on central processing of tonic pain - a pilot study using standardized low-resolution brain electromagnetic tomography (sLORETA). Sci Rep 9(1):6925. https://doi.org/10. 1038/s41598-019-42984-3

48. Northon S, Rustamov N, Piché M (2019) Cortical integration of bilateral nociceptive signals: when more is less. Pain 160(3):724-733. https://doi. org/10.1097/j.pain.0000000000001451

49. Page I, Biner E, Descarreaux M (2018) Vertebral displacements and muscle activity during manual therapy: distinct behaviors between spinal manipulation and mobilization. J Manipulative Physiol Ther 41(9):753761. https://doi.org/10.1016/j.jmpt.2018.07.004
50. Penza CW, Horn ME, George SZ, Bishop MD (2017) Comparison of 2 lumbar manual therapies on temporal summation of pain in healthy volunteers. J Pain 18(11):1397-1408. https://doi.org/10.1016/j.jpain.2017. 07.007

51. Perchet C, Godinho F, Mazza S, Frot M, Legrain V, Magnin M, Garcia-Larrea $L$ (2008) Evoked potentials to nociceptive stimuli delivered by $\mathrm{CO} 2$ or Nd:YAP lasers. Clin Neurophysiol 119(11):2615-2622. https://doi.org/10. 1016/j.clinph.2008.06.021

52. Pfurtscheller G, Lopes da Silva FH (1999) Event-related EEG/MEG synchronization and desynchronization: basic principles. Clin Neurophysiol 110(11):1842-1857. https://doi.org/10.1016/s1388-2457(99)00141-8

53. Pickar JG (2002) Neurophysiological effects of spinal manipulation. Spine J 2(5):357-371. https://doi.org/10.1016/s1529-9430(02)00400-x

54. Pickar JG, Bolton PS (2012) Spinal manipulative therapy and somatosensory activation. J Electromyogr Kinesiol 22(5):785-794. https://doi.org/10. 1016/j.jelekin.2012.01.015

55. Plaghki L, Mouraux A (2003) How do we selectively activate skin nociceptors with a high power infrared laser? Physiology and biophysics of laser stimulation. Neurophysiol Clin 33(6):269-277

56. Ploner M, Sorg C, Gross J (2017) Brain rhythms of pain. Trends Cogn Sci 21(2):100-110. https://doi.org/10.1016/j.tics.2016.12.001

57. Puhl AA, Reinhart CJ, Doan JB, Vernon H (2017) The quality of placebos used in randomized, controlled trials of lumbar and pelvic joint thrust manipulation-a systematic review. Spine J 17(3):445-456. https://doi.org/ 10.1016/j.spinee.2016.11.003

58. Qaseem A, Wilt TJ, McLean RM, Forciea MA (2017) Noninvasive treatments for acute, subacute, and chronic low back pain: a clinical practice guideline from the American College of Physicians. Ann Intern Med 166(7):514-530. https://doi.org/10.7326/M16-2367

59. Qiu Y, Inui K, Wang X, Tran TD, Kakigi R (2001) Conduction velocity of the spinothalamic tract in humans as assessed by $\mathrm{CO} 2$ laser stimulation of C-fibers. Neurosci Lett 311(3):181-184. https://doi.org/10.1016/S03043940(01)02170-X

60. Randoll C, Gagnon-Normandin V, Tessier J, Bois S, Rustamov N, O'Shaughnessy J, Descarreaux M, Piché M (2017) The mechanism of back pain relief by spinal manipulation relies on decreased temporal summation of pain. Neuroscience 349:220-228. https://doi.org/10.1016/j.neuro science.2017.03.006

61. Ronga I, Valentini E, Mouraux A, lannetti GD (2013) Novelty is not enough: laser-evoked potentials are determined by stimulus saliency, not absolute novelty. J Neurophysiol 109(3):692-701. https://doi.org/10.1152/jn.00464. 2012

62. Stochkendahl MJ, Kjaer P, Hartvigsen J, Kongsted A, Aaboe J, Andersen M, Andersen M $\varnothing$, Fournier G, Højgaard B, Jensen MB, Jensen LD (2018) National Clinical Guidelines for non-surgical treatment of patients with recent onset low back pain or lumbar radiculopathy. Eur Spine J 27(1):60-75. https://doi.org/10.1007/s00586-017-5099-2

63. Traeger AC, Buchbinder R, Elshaug AG, Croft PR, Maher CG (2019) Care for low back pain: can health systems deliver? Bull World Health Organ 97(6):423-433. https://doi.org/10.2471/blt.18.226050

64. Triano JJ, Giuliano D, Kanga I, Starmer D, Brazeau J, Screaton CE, Semple C (2015) Consistency and malleability of manipulation performance in experienced clinicians: a pre-post experimental design. J Manipulative Physiol Ther 38(6):407-415. https://doi.org/10.1016/j.jmpt.2015.05.002

65. Wirth B, Gassner A, de Bruin ED, Axen I, Swanenburg J, Humphreys BK, Schweinhardt P (2019) Neurophysiological effects of high velocity and low amplitude spinal manipulation in symptomatic and asymptomatic humans: a systematic literature review. Spine 44(15):E914-E926. https:// doi.org/10.1097/BRS.0000000000003013

\section{Publisher's Note}

Springer Nature remains neutral with regard to jurisdictional claims in published maps and institutional affiliations. 